\title{
Rethinking the extrinsic incubation period of malaria parasites
}

\author{
Johanna R. Ohm ${ }^{1 *}$, Francesco Baldini ${ }^{2}$, Priscille Barreaux ${ }^{1}$, Thierry Lefevre ${ }^{3}$, Penelope A. Lynch ${ }^{4}$, Eunho Suh ${ }^{1}$, \\ Shelley A. Whitehead' and Matthew B. Thomas ${ }^{1}$
}

\begin{abstract}
The time it takes for malaria parasites to develop within a mosquito, and become transmissible, is known as the extrinsic incubation period, or EIP. EIP is a key parameter influencing transmission intensity as it combines with mosquito mortality rate and competence to determine the number of mosquitoes that ultimately become infectious. In spite of its epidemiological significance, data on EIP are scant. Current approaches to estimate EIP are largely based on temperature-dependent models developed from data collected on parasite development within a single mosquito species in the 1930s. These models assume that the only factor affecting EIP is mean environmental temperature. Here, we review evidence to suggest that in addition to mean temperature, EIP is likely influenced by genetic diversity of the vector, diversity of the parasite, and variation in a range of biotic and abiotic factors that affect mosquito condition. We further demonstrate that the classic approach of measuring EIP as the time at which mosquitoes first become infectious likely misrepresents EIP for a mosquito population. We argue for a better understanding of EIP to improve models of transmission, refine predictions of the possible impacts of climate change, and determine the potential evolutionary responses of malaria parasites to current and future mosquito control tools.
\end{abstract}

Keywords: Malaria, Mosquito, Extrinsic incubation period, EIP, Temperature

\section{Background}

The extrinsic incubation period (EIP) of malaria, also called the period of sporogony, describes the time it takes for parasites to develop in the mosquito from point of ingestion via an infected blood meal, through to the point at which sporozoites enter the salivary glands and the mosquito becomes infectious. In the classic models of malaria transmission (e.g. [1-4]), the EIP is one of the most influential parameters because it interacts with adult mosquito survival rate as an exponential term, meaning that even very small changes in EIP can have a large effect on the number of mosquitoes living long enough to be able to transmit parasites. Changes in EIP potentially have much greater impact than equivalent changes in traits such as vector competence (i.e. the ability of vectors to become infectious) or vector density. Despite its epidemiological importance, EIP remains poorly characterized.

\footnotetext{
*Correspondence: jo.ohm@psu.edu

${ }^{1}$ Center for Infectious Disease Dynamics, Pennsylvania State University, University Park, PA, USA

Full list of author information is available at the end of the article
}

Our current understanding of EIP derives largely from research conducted in the early to mid-1900s, wherein the development times of the human malaria parasites Plasmodium falciparum, $P$. vivax and $P$. malariae were examined in the mosquito Anopheles maculipennis across a range of constant temperatures [5]. These data were used to construct degree-day models to predict the EIP of malaria parasites at a given environmental temperature $[1,2]$. The models assume it takes a set number of accumulated degree days (DD) for malaria parasites to complete their development once mean daily temperature ( $\mathrm{T}$, in degrees Celsius) exceeds a lower temperature threshold for development $\left(\mathrm{T}_{\mathrm{min}}\right)$. For P. falciparum, $\mathrm{DD}=111$ and $\mathrm{T}_{\min }=16{ }^{\circ} \mathrm{C}$, giving EIP $=111 /(\mathrm{T}-16)$ [1]. For $P$. vivax the equivalent is EIP $=105 /(\mathrm{T}-14.5)$, and for $P$. malariae $\mathrm{EIP}=144 /(\mathrm{T}-16)[1]$.

The Detinova degree-day models of $P$. falciparum and $P$. vivax described above have become lore [1]. Many contemporary studies that provide an estimate of EIP do so without acknowledging the source, let alone attempting any direct validation. However, in spite of their widespread use, the assumptions underpinning these models 
have received little attention. For instance, Nikolaev [5] defined EIP as the time at which sporozoites were first observed in the salivary glands of an individual infected mosquito, yet whether this is the most relevant measure in terms of overall transmission potential of a mosquito population is not clear. The degree-day models also assume that EIP can be estimated using mean temperatures alone. Whether other factors, such as parasite and vector genetics, or other sources of environmental variation, also play a role has been virtually ignored. Equally, whether there is a genetic basis for variation in EIP and potential for evolution in parasite development rate under different environmental conditions (e.g. in response to vector control interventions or climate change) is unknown. Our aim in the current paper is to examine these assumptions in order to improve our understanding of EIP, identify key knowledge gaps, and motivate further work to better characterize EIP moving forward.

\section{What factors determine the EIP?}

The influence of temperature

The original work of Nikolaev evaluated EIP of different malaria species across a range of constant temperatures (from $19-20{ }^{\circ} \mathrm{C}$ to $30{ }^{\circ} \mathrm{C}$ for P. falciparum, and $15-16{ }^{\circ} \mathrm{C}$ to $30{ }^{\circ} \mathrm{C}$ for $P$. vivax) [5]. In nature, however, mosquitoes do not live at a constant temperature but experience daily temperature fluctuations. There is now significant literature indicating that daily temperature variation can have a substantial effect on mosquito and parasite life history, beyond the effects of mean temperature [6-13]. In particular, theory and empirical evidence indicate that daily temperature fluctuations are likely to have the greatest influence toward the upper and lower thermal limits, with daily variation acting to increase parasite development rate under low mean temperatures, slowing development rate under high mean temperatures, and potentially having no net effect under intermediate conditions $[6,14,15]$. Thus, estimates of EIP derived under constant temperatures may not reflect the actual EIPs occurring in nature.

We are aware of no explicit empirical tests of how daily temperature variation impacts EIP of human malaria parasites. However, studies using a rodent malaria confirm the contrasting effects of daily temperature variation on parasite development rate under cool and warm conditions [6], so it seems likely that EIP of human malaria parasites could be similarly affected. Studies on dengue virus development within Aedes aegypti also show that temperature fluctuations shorten EIP under cool mean temperatures, but that fluctuations have no effect on virus development rate at higher temperatures, despite reduced vector competence [13].

Current degree-day models also define a lower temperature threshold below which development ceases. For P. falciparum $\mathrm{T}_{\min }=16{ }^{\circ} \mathrm{C}$. However, the lowest temperature measured for $P$. falciparum in the studies of Nikolaev was $19-20{ }^{\circ} \mathrm{C}$ [5], and $\mathrm{T}_{\min }=16{ }^{\circ} \mathrm{C}$ was selected by Detinova [1] based on the earlier work of Moshkovsky [2] who fitted a linear regression to the parasite development rate data of Nikolaev and found the line crossed the $\mathrm{x}$-axis at $16{ }^{\circ} \mathrm{C}$. Other studies provide varied estimates of the lower thermal thresholds of human malaria, ranging from as low as $15^{\circ} \mathrm{C}$ to as high as 24-26 ${ }^{\circ} \mathrm{C}$ for P. falciparum, and from $14.5^{\circ} \mathrm{C}$ to $17.5{ }^{\circ} \mathrm{C}$ for $P$. vivax (see [16] and Table 1.3 in [17]). Few studies attempt to estimate EIP of $P$. falciparum at temperatures below $20^{\circ} \mathrm{C}$ (Fig. 1). Thus, whether we can define $16{ }^{\circ} \mathrm{C}$ as the appropriate lower developmental threshold as used in the Detinova model [1] is currently unknown. This lack of knowledge is striking given that $\mathrm{T}_{\text {min }}$ is so integral to the degree-day model approach.

Additionally, the degree-day models assume the relationship between parasite development rate (the reciprocal of the EIP) and temperature is linear $[1,2]$. In contrast, a number of recent theoretical studies describe malaria parasite development rate as a unimodal, nonlinear function [18-21]. Which of these approaches is most appropriate depends critically on whether there is an optimum temperature for development and whether the rate declines as temperatures increase above this optimum. Unfortunately, current evidence is again limited. The studies adopting unimodal functions include data from a very limited number of historic studies

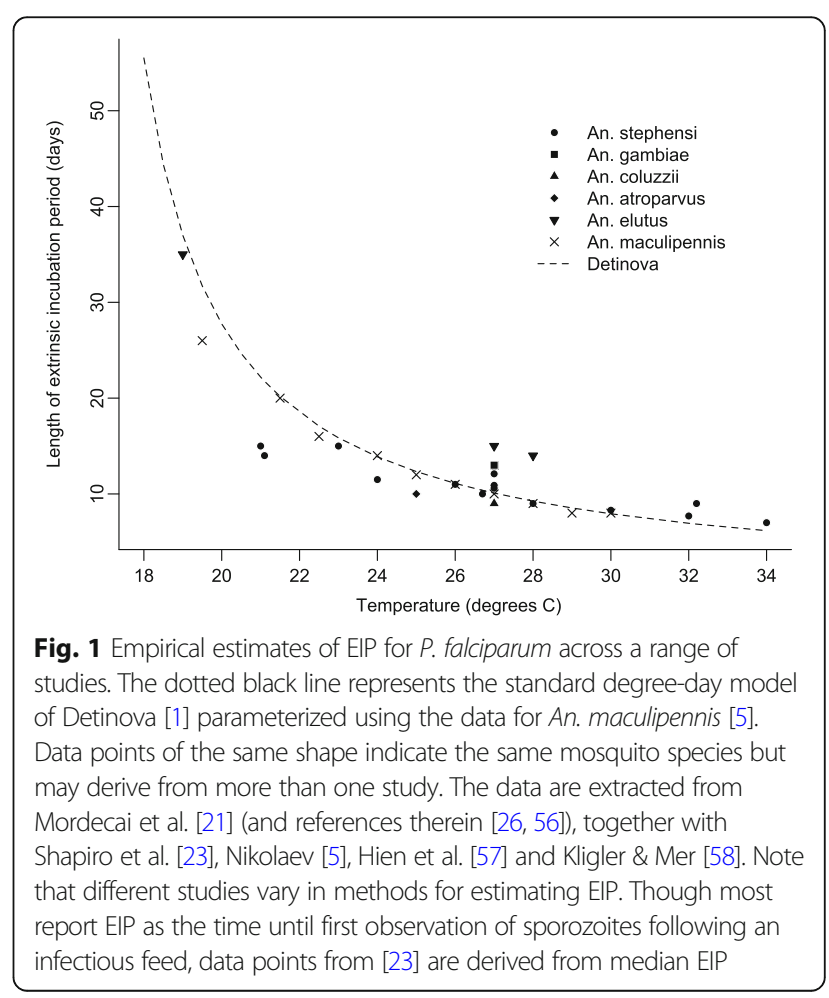


where dissection of mosquitoes at high temperatures revealed no sporozoites in the salivary glands (e.g. [22]). However, the absence of sporozoites does not necessarily equate to zero growth rate. This distinction is not simply semantic; we ought to know whether high temperatures limit transmission because of a decline in vector competence (which could be direct parasite mortality or perhaps mediated via the mosquito), or because parasite growth slows and EIP becomes progressively longer. In the recent study of Shapiro et al. [23] there was no evidence of a non-linear decline in parasite development rate up to $34{ }^{\circ} \mathrm{C}$, even though the proportion of mosquitoes becoming infectious declined at temperatures above $27{ }^{\circ} \mathrm{C}$. More data are needed to resolve this fundamental issue.

\section{Parasite genetic diversity}

Nikolaev's study [5] identified differences in the EIP between three Plasmodium species. Other studies have further demonstrated interspecific variation in EIP [24-26]. The genetic basis for these differences in EIP is poorly understood. Additionally, whether there is intraspecific variation in EIP between parasite genotypes is unknown. The Detinova degree-day models assume no intraspecific variation but we are not aware of any empirical studies investigating this assumption.

Studies from other vector-borne pathogens provide some evidence of intraspecific variation in EIP. For example, the emergence of a new dominant genotype of West Nile virus in North America has been attributed to the new genotype having a shorter EIP in Culex mosquito vectors compared to the original strain [27]. Similarly, the Southeast Asian genotype of dengue serotype 2 virus has displaced the American genotype in several countries [28] which has been explained by its shorter EIP resulting in an estimated 2- to 65 -fold increase in the vectorial capacity of the Ae. aegypti vector [29]. Additionally, differences in dissemination rate of three strains of dengue serotype 2 viruses within the same $A e$. aegypti colony have also been observed [30], further suggesting that the pathogen's intraspecific variation in EIP is genetically influenced. However, intraspecific variation in EIP is not always observed among viruses. A single mutation between two isolates of chikungunya virus (CHIKV) favored transmissibility by Aedes albopictus [31] and has been associated with an outbreak that occurred in Indian Ocean territories, but no quantitative differences in EIPs between these strains were observed [32]. In addition, a statistical analysis aimed at estimating the relationship between temperature and EIP in three orbiviruses transmitted by Culicoides biting midges showed that the rate of virus replication was mostly consistent among the different pathogen genotypes [33].
Given the high levels of genetic variation within malaria parasite species [34-38], it seems likely that there could be genotypic variation for EIP [39]. Different Plasmodium genotypes have been shown to vary in their capacity to infect a specific mosquito species [40, 41], possibly due to different immune evasion mechanisms [42]. Additionally, parasite growth rates within the vertebrate host are under genetic control [39, 42]. Better characterizing intraspecific variation in sporogony could improve investigation of local transmission dynamics (e.g. [43]) and could help in understanding the spread of drug resistant genotypes (cf. [44, 45]).

\section{Vector genetic diversity}

There are approximately 70 species of mosquitoes in the genus Anopheles known to contribute to transmission of malaria parasites to humans [46]. The current degreeday models of EIP were derived from studies on one population of a single species, the Eurasian vector Anopheles maculipennis [5]. Few researchers would be happy to accept that all populations or species of Anopheles mosquitoes are equally permissive to malaria infection, and there has been substantial research investment to understand the genetic mechanisms underlying variation in susceptibility/refractoriness (e.g. [36, 47, 48]). Yet for EIP the prevailing assumption is that all vector species and populations are identical and the EIP is a property of the parasite response to mean temperature alone. Indeed, White \& Rao [49] state "for lack of any evidence to the contrary, it must be assumed that differences in vector species does $[$ sic] not affect the results [of EIP]".

In Fig. 1 we present all the available data we can find from studies that have explicitly measured EIP of $P$. falciparum (note that we followed the approach of Mordecai et al. [21] and excluded studies if they did not demonstrate adequate control of temperature, were unclear on parasite species, or had insufficient sample size such as reporting infections from dissection of single mosquitoes). The figure reveals that data are extremely sparse and that certain empirical estimates of EIP do not clearly match the standard degree-day model. Whether there are significant differences between vector species is impossible to say as there are insufficient data to generate species-specific EIP models for any of the key malaria vectors in Africa, Asia or Latin America.

In addition to the potential for interspecific differences in EIP between vectors (Fig. 1), there is the potential for intraspecific variation. In a recent study, Ye et al. [50] examined EIP of dengue across 40 genetically distinct families of Aedes aegypti. They showed significant differences in EIP (measured as time to detectable virus in the saliva) between families ranging from 4-14 days, and that variation in EIP was highly heritable ( 40\%). 
Shorter EIPs were additionally correlated with shorter vector lifespans and higher virulence. This work demonstrates that EIP of dengue is largely controlled by variation in the mosquito genome. We are aware of no studies on malaria vectors examining intraspecific genetic variation in EIP. The data from Shapiro et al. [23] indicate differences between individual mosquitoes but the mechanisms are unclear. However, with evidence for genetic influence on other aspects of malaria parasite infection such as resistance/susceptibility [35, 47, 48, 51], interactions with insecticide resistance [52], and vector genotype $\times$ parasite genotype interactions $[40,53,54]$, it would be surprising if there was no influence of mosquito genetics on EIP.

\section{Other biotic and abiotic factors}

The complex interplay between parasite and vector traits that determine overall transmission can be influenced by many factors [55-58]. Larval food limitation has been shown to decrease malaria parasite survival [59] and affect infection prevalence and intensity [60, 61]. The mechanisms behind these observations are not well understood but could be linked to altered immune response, resource allocation within the vector [59-61], or effects on adult body size that influence the blood meal volume and hence the number of infecting parasites (note that temperatures in the larval environment also impact ultimate adult size [62]). Importantly, quality of the larval habitat has been shown to affect EIP for both dengue [63] and P. falciparum [23] independent of temperature.

Food intake by adult mosquitoes can also affect parasite development. Relatively few studies have looked at the impact of sugar feeding on mosquito or parasite life history but there is evidence that nectar from different plants can potentially inhibit or enhance parasite load and rate of parasite development [57, 64]. Blood-feeding has also recently been shown to influence EIP of dengue virus in Aedes mosquitoes, with additional blood meals accelerating virus development [65].

Malaria parasites potentially compete with many organisms inside mosquitoes [66-69], including mixed infections with other malaria parasite genotypes [70, 71]. These interactions can impact parasite establishment and density via competition or immune-mediate mechanisms [72]. What effect they might have on EIP of malaria parasites is not known, but for dengue, the presence of an intracellular bacterial parasite (Wolbachia) has been shown to extend the EIP [73, 74]. There is further potential for parasite/pathogen-mediated effects via trans-generational immune priming, which can confer lasting protection within an individual [75] and in its offspring [76]. If parental exposure to parasites has consequences for malaria parasite resistance in the offspring
[76], it is possible this could impact EIP, though this has not yet been explored.

\section{How should EIP be measured?}

The original work of Nikolaev [5] dissected mosquitoes at various time points following an infectious blood meal and defined EIP as the time at which sporozoites were first observed in mosquito salivary glands. Capturing the time of the first mosquitoes to become infectious might make sense: mosquitoes that allow rapid parasite migration through their bodies have more opportunities to infect humans at subsequent bites and so might be the most epidemiological relevant individuals. Equally, if parasite development is highly synchronized between individuals, then the time to first infection will likely be a reasonable approximation for the mosquito population as a whole. On the other hand, if development is widely distributed between mosquitoes, then a few early infectious mosquitoes might be unrepresentative of the total mosquito population, and be a poor predictor for force of infection.

A number of recent empirical studies (e.g. [23, 57]) have demonstrated that the time parasites take to reach the salivary glands is not the same for all mosquitoes in a population, even if they have received the same infectious blood meal and are maintained under identical conditions, again highlighting the need to understand sources of this variation [54]. Furthermore, variance in EIP and the median EIP value are affected by temperature [23]. Under warm conditions the median is shorter and there is less variation in duration of sporogony between mosquitoes, but as conditions cool, the median increases and the time between the first and last mosquitoes to become infectious can extend to several days, widening the distribution of EIP [23].

In Additional file 1, we present an outline of a model developed to examine whether different measures of EIP affect estimates of the probability that mosquitoes live long enough to become infectious. We based our analysis on the study of Shapiro et al. [23], which measured the EIP of P. falciparum in An. stephensi across six constant temperatures ranging from $21-34{ }^{\circ} \mathrm{C}$. Briefly, the dynamics of sporogony were characterized by a logistic function (Fig. 2, Additional file 1), which enables us either to define individual measures of EIP (the 10percentile, 50-percentile or 90-percentile), or to represent the full growth kinetics of parasites across the mosquito population.

In order to examine the proportion of infected mosquitoes that survive through the different measures of EIP we needed to estimate adult mosquito mortality rate. Many transmission models assume a constant daily mortality rate. In Fig. 3a we weight the proportion of mosquitoes that developed sporozoites at each temperature 


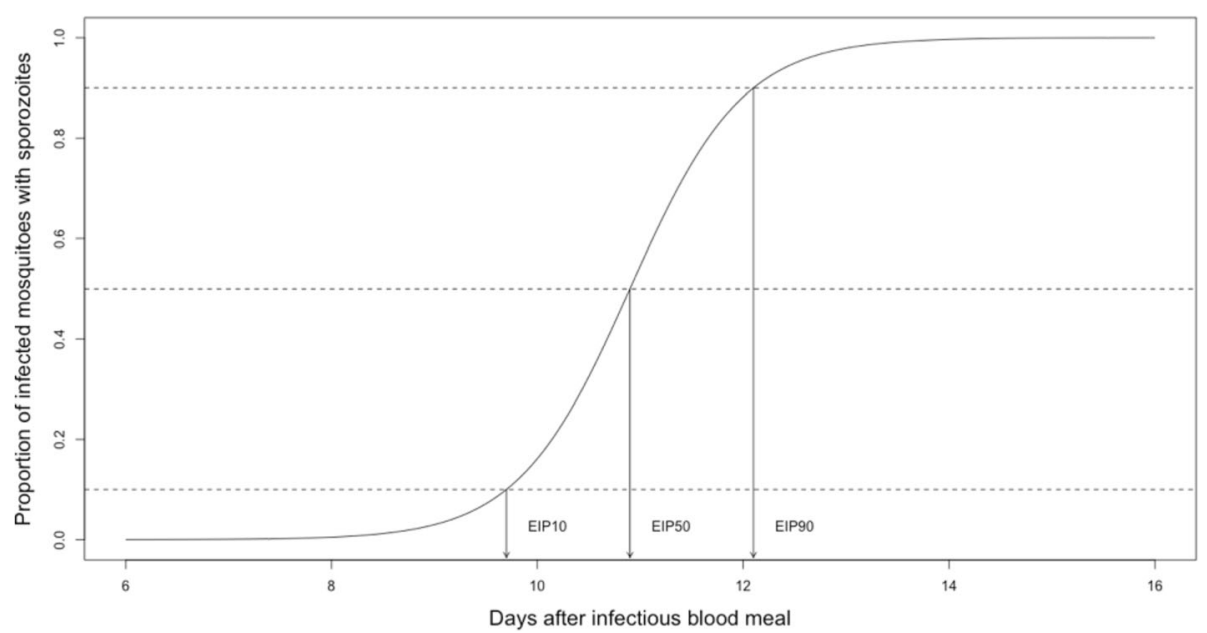

Fig. 2 Proportion of malaria-infected mosquitoes with sporozoites present in the salivary glands (i.e. becoming infectious) over time following an infectious blood meal. Here the dynamics of EIP are characterized using a logistic model following the approach of Paaijmans et al. [77] and Shapiro et al. [23, 60] (and see also data in Hien et al. [57]). The conventional way of estimating EIP is to measure the time at which sporozoites first appear in salivary glands of infected mosquitoes (approximating the EIP 10 ). However, given EIP is not perfectly synchronized between individual mosquitoes, the EIP could equally be characterized using alternative measures such as the median value for the mosquito population (EIP ${ }_{50}$ ), or the time at which the maximum proportion of the population become infectious (approximating the EIP ${ }_{90}$ ). In this illustrative example we assume all infected mosquitoes go on to become infectious. If conversion efficiency of oocysts to sporozoites is less than $100 \%$, the asymptote will be reduced

by the proportion that survived through sporogony for a constant mortality rate of $10 \%$ per day, comparing the $\mathrm{EIP}_{10}, \mathrm{EIP}_{50}, \mathrm{EIP}_{90}$, the standard degree-day model, and the full logistic model (Additional file 1: Table S1). In Fig. $3 \mathrm{~b}$ we conduct a similar analysis but rather than assume a constant daily mortality rate we used the actual temperature-dependent mortality rates measured by Shapiro et al. [23] for each of the six temperatures (Additional file 1: Table S2).

Comparison of Fig. 3a with $3 \mathrm{~b}$ shows that the pattern of adult mortality has a qualitative effect on the proportion of infected mosquitoes predicted to be alive and infectious for our different measures of EIP. With constant daily mortality rate there is a general trend for the proportion of infectious mosquitoes to increase as temperatures rise, since warmer temperatures shorten EIP whichever way it is characterized. With temperaturedependent mortality, however, the proportion of infectious mosquitoes tends to fall as temperature extends beyond $27{ }^{\circ} \mathrm{C}$, since reductions in EIP are offset by increases in daily mosquito mortality rates at higher temperatures.

In addition, regardless of how mortality is estimated, at low temperatures the standard degree-day model tends to underestimate the probability of mosquitoes being alive and infectious compared to the estimates based on the empirical data of Shapiro et al. [23]. This difference largely derives from the fact that Shapiro et al. [23] reported more rapid sporogony than Nikolaev [5] at cooler temperatures. At temperatures above $27{ }^{\circ} \mathrm{C}$ the degree-day model increasingly approximates the $\mathrm{EIP}_{10}$, which is to be expected as Nikolaev [5] estimated EIP from the first few mosquitoes to become infectious (which is close to the $\mathrm{EIP}_{10}$ ) and the data of Shapiro et al. [23] and Nikolaev [5] are more similar at high temperatures. Perhaps most important is that the EIP $_{50}$ yields almost identical values to approximations based on the full logistic model, while the $\mathrm{EIP}_{10}$ and $\mathrm{EIP}_{90}$ tend to over and under estimate the probability of a mosquito being alive and infectious, respectively. This result indicates that it is important to characterize the full dynamics of sporogony and that the distribution of EIP is better estimated using the median $\operatorname{EIP}\left(\mathrm{EIP}_{50}\right)$, rather than beginning or end points of the distribution. This is not how EIP has been interpreted for almost a century.

\section{Conclusions}

Current understanding of EIP of malaria parasites is limited. There are very few empirical data and those that exist tend to report EIP inappropriately. Moreover, basic information regarding the genetic and environmental determinants of EIP is lacking. This is unfortunate as the potential environmental and genetic influences are numerous and likely to have profound evolutionary and epidemiological implications [77-83]. One obvious implication is that the intensity of malaria transmission will vary spatially and temporally depending on environmental fluctuations and specific vector-parasite combinations. It could be that effect sizes are small and that the established degree-day models capture the variation in 


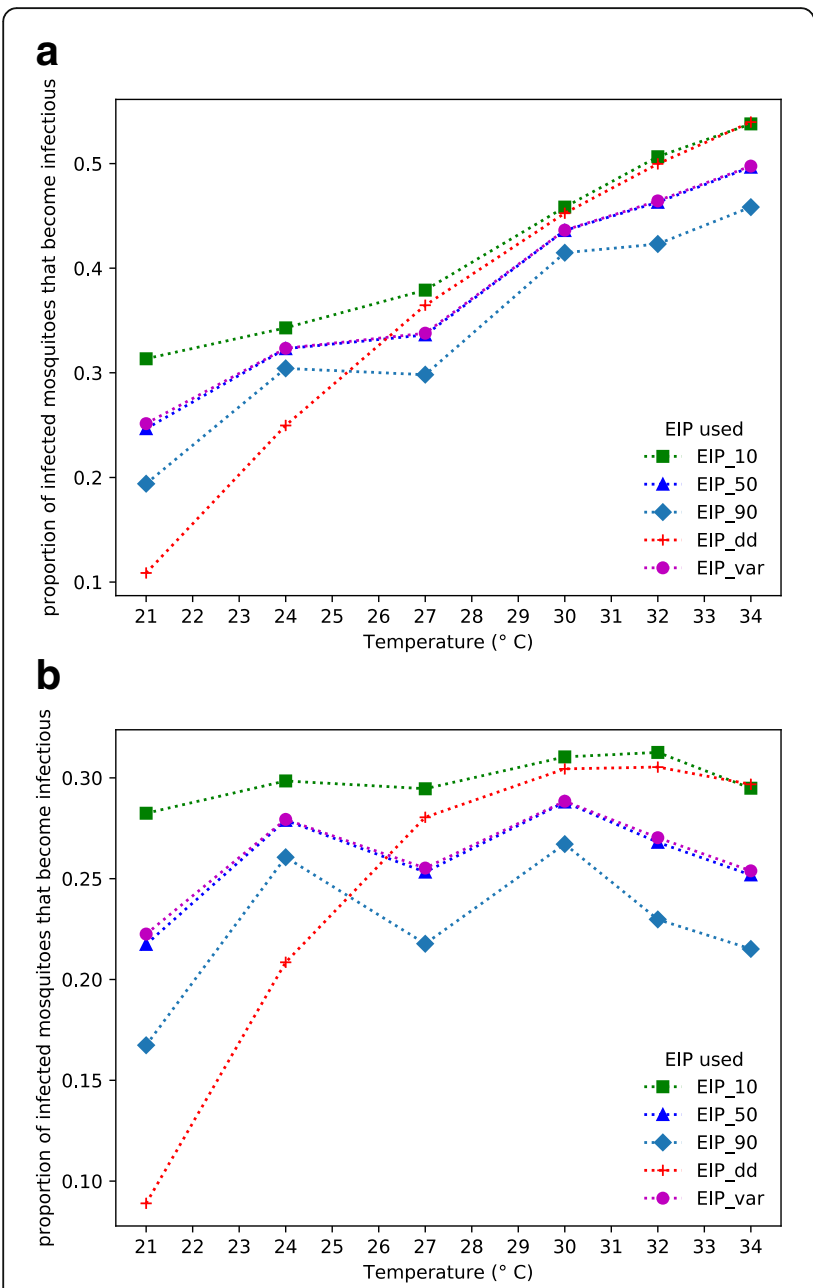

Fig. 3 The proportion of infected mosquitoes predicted to survive the duration of EIP and be able to transmit $P$. falciparum parasites at different temperatures. The EIP var values refer to the full logistic models describing the dynamics of sporogony across six constant temperatures presented in Shapiro et al. [23]. The EIP 10, EIP $_{50}$ and EIP $_{90}$ values represent the 10-, 50- and 90-percentile points from the logistic curves. The EIP dd values are from the classic Detinova degree-day model [1]. a Assumes a constant mortality rate of adult mosquitoes of $10 \%$ per day. $\mathbf{b}$ Assumes adult mortality rate to vary with temperature based on the data presented in Shapiro et al. [23]

EIP across time and space adequately. However, it could also be that mosquito species, mosquito condition, parasite strain, etc. have a substantial influence. This should not be an open question. There has been considerable speculation regarding possible impacts of climate warming on malaria transmission $[19,21,81,83]$, yet the effects could depend as much on the specifics of the local mosquito-parasite pairing as the absolute change in temperature itself. More empirical studies are required to rigorously examine EIP both as a stand-alone trait, and in the context of other essential components of vectorial capacity, such as mosquito density, adult longevity, and biting rate, which all contribute to overall transmission. Such studies would be facilitated greatly by the optimization of non-destructive methodologies allowing fine temporal resolution of EIP within individual mosquitoes, as is now possible for arboviruses [50, 84, 85]. In terms of transmission dynamics, it would also be valuable to determine the parasite's ability to adjust its development rate in response to environmental cues (adaptive phenotypic plasticity). For instance, can malaria parasites adaptively speed up their EIP when their transmission is compromised by the imminent death of their vectors (perhaps in old mosquitoes, those exposed to insecticides, or in the presence of competing parasites)? In a related way, given transmission is ultimately dependent on the bite of an infectious mosquito, it would be interesting to explore whether EIP could potentially be linked to biting rate and gonotrophic cycle. Like EIP, biting behavior is influenced by a suite of environmental factors $[86,87]$ and it is possible that the duration of EIP is rhythmically modulated to avoid the situation where the parasite is ready to be transmitted but the mosquito is not ready to feed, either because the mosquito is in the middle of a gonotrophic cycle [7] or because it is physiologically constrained [87, 88]. Such condition-dependent developmental strategies have been described in blood-stage malaria parasites [89, 90] and deserve considerations in infected mosquitoes. Finally, understanding the extent to which EIP is genetically variable is also crucial to understanding the capacity of EIP to evolve in response to malaria interventions or mosquito life history, as genetic variation fuels evolution. Current core vector control tools (long-lasting insecticide-treated bed nets (LLINs) and indoor residual insecticide sprays (IRS)) act, in part, by changing mosquito population age structure [91, 92]. These tools exploit the fact that the EIP is long relative to the lifespan of most mosquitoes, and that mosquitoes take multiple blood meals throughout their lifetime. By increasing the probability of mortality per blood feeding event, LLINs and IRS reduce the number of mosquitoes that live long enough for the parasite to complete EIP. Other prospective control tools also target the 'old infectious' mosquitoes [91, 92]. There is now a substantial industry built around understanding and managing the evolutionary responses of mosquitoes to insecticides and other vector control tools (e.g. see [93]). Whether vector control tools can drive evolutionary changes in EIP and select for parasite clones with shorter EIPs is unknown but should, perhaps, become part of an extended insecticide-resistance monitoring process. The fitness of parasites should increase with shorter EIP, unless faster developing parasites inflict higher mortality costs on mosquitoes or come with fitness trade-offs to the parasite such as reduced infectivity (as discussed in [54]). Whether mosquito fitness is affected by EIP length of malaria 
parasites is unknown. The implications for transmission could depend on relationships with relevant transmission traits such as mosquito longevity or parasite load [54]. For example, are fast developing clones also those that are the most virulent and reduce mosquito longevity? Are fast developing parasites also those that produce the fewest transmissible stages? These potential trade-offs and constraints may have important implications for understanding the evolutionary potential of EIP. More broadly, the effects of parasite drug resistance and mosquito insecticide resistance - two important sources of genetic variation - on EIP deserve attention.

\section{Additional file}

Additional file 1: Text. Numerical approximation of proportion of vectors surviving to become infectious assuming logistic EIP. Table S1. Comparison of results for approximation of probability of surviving from infection to infectiousness using logistic model. Results used for plots in main text are highlighted. Chosen $D_{\max }$ and $\delta$ give results consistent to $6 \mathrm{dp}$ with results from ten times smaller $\delta$ and $D_{\max }$ of $100 \mathrm{vs} 30$, indicating that for the intended purpose, no material benefit would be gained from using smaller $\delta$ or larger $D_{\max }$. Table $\mathbf{S 2}$. The temperature-related values used for $k$, tM, and $\mu$, taken from Shapiro et al [23]. (DOC 68 kb)

\section{Abbreviations}

EIP: Extrinsic incubation period; EIP 10 : Extrinsic incubation period measured as the time until 10 percent of infected mosquitoes become infectious; EIP $_{50}$ : Extrinsic incubation period measured as the time until 50 percent of infected mosquitoes become infectious which equates to the median time of sporozoite development; EIP 90 : Extrinsic incubation period measured as the time until 90 percent of infected mosquitoes become infectious

\section{Acknowledgements}

The development of the ideas presented in this review were prompted by discussion held at the Vector BiTE Research Collaboration Network meeting at Imperial College London, 2017.

\section{Funding}

MBT, JRO, PL, SAW, ES and PB were supported in part by the National Science Foundation (DEB-1518681) and the National Institutes of Health (NIH NIAID R01Al110793). FB was supported by EMBO ALTF 43-2014 and AXA RF 14-AXA-PDOC-130 and TL was supported by ANR grant 16-CE35-0007. The funders had no role in study design, data collection and analysis, decision to publish, or preparation of the manuscript.

\section{Availability of data and materials}

All data analyzed and statistical methods used are included in this review or cited in the references.

\section{Authors' contributions}

$M B T, J R O$ and $T L$ originally conceived of the idea for a manuscript to reevaluate how EIP is defined through the VectorBiTE EIP working group. PL constructed figures and models to demonstrate quantitative examples for how EIP estimates vary. SAW, ES, FB and PB developed ideas with MBT, JRO and TL at the 2017 VectorBiTE EIP working group meeting and assisted in writing and editing the manuscript. All authors read and approved the final manuscript.

\section{Ethics approval and consent to participate}

Not applicable

\section{Consent for publication}

Not applicable

\section{Competing interests}

The authors declare that they have no competing interests.

\section{Publisher's Note}

Springer Nature remains neutral with regard to jurisdictional claims in published maps and institutional affiliations.

\section{Author details}

${ }^{1}$ Center for Infectious Disease Dynamics, Pennsylvania State University, University Park, PA, USA. ${ }^{2}$ Institute of Biodiversity Animal Health and Comparative Medicine, University of Glasgow, Glasgow, Scotland, UK. ${ }^{3}$ MIVEGEC, IRD, CNRS, University of Montpellier, Montpellier, France. ${ }^{4}$ College of Life and Environmental Sciences, Penryn Campus, University of Exeter, Cornwall, UK.

Received: 18 December 2017 Accepted: 28 February 2018

Published online: 12 March 2018

\section{References}

1. Detinova TS. Age-grouping methods in Diptera of medical importance with special reference to some vectors of malaria. Monogr Ser World Health Organ. 1962:47:13-91.

2. Moshkovsky SD. On the dependency of the speed of development of plasmodia malaria on temperature. Med Para Para Bol. 1946;15:19-32.

3. Garrett-Jones C, Shidrawi GR. Malaria vectorial capacity of a population of Anopheles gambiae: an exercise in epidemiological entomology. Bull World Health Organ. 1969;40(4):531-45.

4. Smith DL, McKenzie FE. Statics and dynamics of malaria infection in Anopheles mosquitoes. Malar J. 2004;3:13.

5. Nikolaev BP. On the influence of temperature on the development of malaria plasmodia inside the mosquito. Trans Pasteur Inst Epi Bact Leningrad. 1935;2:1-5

6. Paaijmans KP, Blanford S, Bell AS, Blanford JI, Read AF, Thomas MB. Influence of climate on malaria transmission depends on daily temperature variation. Proc Natl Acad Sci USA. 2010;107(34):15135-9.

7. Paaijmans KP, Cator $L$, Thomas MB. Temperature-dependent pre-bloodmeal period and temperature-driven asynchrony between parasite development and mosquito biting rate reduce malaria transmission intensity. PLoS One. 2013:8(1):e55777.

8. Paaijmans KP, Heinig RL, Seliga RA, Blanford Jl, Blanford S, Murdock CC, et al Temperature variation makes ectotherms more sensitive to climate change. Global Change Biol. 2013;19(8):2373-80.

9. Murdock CC, Moller-Jacobs LL, Thomas MB. Complex environmental drivers of immunity and resistance in malaria mosquitoes. Proc Biol Sci. 2013; 280(1770):20132030

10. Murdock CC, Sternberg ED, Thomas MB. Malaria transmission potential could be reduced with current and future climate change. Sci Rep. 2016;6(1):27771.

11. Beck-Johnson LM, Nelson WA, Paaijmans KP, Read AF, Thomas MB, Bjørnstad ON. The importance of temperature fluctuations in understanding mosquito population dynamics and malaria risk. Royal Soc Open Sci. 2017; 4(3):160969

12. Lambrechts $L$, Paaijmans KP, Fansiri T, Carrington LB, Kramer LD, Thomas $M B$, et al. Impact of daily temperature fluctuations on dengue virus transmission by Aedes aegypti. Proc Natl Acad Sci USA. 2011;108(18):7460-5.

13. Carrington LB, Armijos MV, Lambrechts L, Scott TW. Fluctuations at a low mean temperature accelerate dengue virus transmission by Aedes aegypti. PLoS Negl Trop Dis. 2013;7(4):e2190.

14. Worner SP. Performance of phenological models under variable temperature regimes: consequences of the Kaufmann or rate summation effect. Environ Entomol. 1992;21(4):689-99.

15. Ruel JJ, Ayres MP. Jensen's inequality predicts effects of environmental variation. Trends Ecol Evol (Amst). 1999;14(9):361-6.

16. Shute PG, Maryon M. Malaria in England past, present and future. R Soc Health J. 1974;94(1):23-9.

17. Hutchinson RA. Mosquito borne diseases in England: past, present and future risks, with special reference to malaria in the Kent Marshes. PhD thesis, Durham University, UK; 2004, 183 pp.

18. Ikemoto T. Tropical malaria does not mean hot environments. J Med Ent. 2008:45(1):963-9.

19. Paaijmans KP, Read AF, Thomas MB. Understanding the link between malaria risk and climate. Proc Natl Acad Sci USA. 2009;106(33):13844-9. 
20. Beck-Johnson LM, Nelson WA, Paaijmans KP, Read AF, Thomas MB, Bjørnstad ON. The effect of temperature on Anopheles mosquito population dynamics and the potential for malaria transmission. PLoS One. 2013:8(11):e79276.

21. Mordecai EA, Paaijmans KP, Johnson LR, Balzer C, Ben-Horin T, de Moor E, et al. Optimal temperature for malaria transmission is dramatically lower than previously predicted. Ecol Lett. 2012;16(1):22-30.

22. Siddons L. Observations on the influence of atmospheric temperature and humidity on the infectivity of Anopheles culicifacies Giles. J Malar Inst India. 1944;5:375-88.

23. Shapiro LLM, Whitehead SA, Thomas MB. Quantifying the effects of temperature on mosquito and parasite traits that determine the transmission potential of human malaria. PLoS Biol. 2017;15(10):e2003489.

24. Collins WE, Sullivan JS, Nace D, Williams T, Sullivan JJ, Galland GG, et al. Experimental infection of Anopheles farauti with different species of Plasmodium. J Parasitol. 2002;88(2):295.

25. Stratman-Thomas WK. The influence of temperature on Plasmodium vivax. Am J Trop Med Hyg. 1940;1-20(5):703-15.

26. Knowles R, Basu BC. Laboratory studies on the infectivity of Anopheles stephensi. J Malar Inst India. 1943;5:1-29.

27. Moudy RM, Meola MA. Morin L-LL, Ebel GD, Kramer LD. A newly emergent genotype of West Nile virus is transmitted earlier and more efficiently by Culex mosquitoes. Am J Trop Med Hyg. 2007;77(2):365-70.

28. Rico-Hesse REA. Origins of dengue type 2 viruses associated with increased pathogenicity in the Americas. Virology. 1997;230(2):244-51.

29. Anderson JR, Rico-Hesse R. Aedes aegypti vectorial capacity is determined by the infecting genotype of dengue virus. Am J Trop Med Hyg. 2006;75(5):886-92.

30. Christofferson RC, Mores CN. Estimating the magnitude and direction of altered arbovirus transmission due to viral phenotype. PLoS One. 2011;6(1):e16298.

31. Schuffenecker I, Iteman I, Michault A, Murri S, Frangeul L, Vaney M-C, et al. Genome microevolution of chikungunya viruses causing the Indian Ocean outbreak. PLoS Med. 2006:3(7):e263.

32. Vazeille M, Moutailler S, Coudrier D, Rousseaux C, Khun H, Huerre M, et al. Two chikungunya isolates from the outbreak of La Reunion (Indian Ocean) exhibit different patterns of infection in the mosquito, Aedes albopictus. PLoS One. 2007:2(11):e1168-9.

33. Carpenter S, Wilson A, Barber J, Veronesi E, Mellor P, Venter G, et al. Temperature dependence of the extrinsic incubation period of orbiviruses in Culicoides biting midges. PLoS One. 2011;6(11):e27987.

34. Jeffares DC, Pain A, Berry A, Cox AV, Stalker J, Ingle CE, et al. Genome variation and evolution of the malaria parasite Plasmodium falciparum. Nat Genet. 2007;39(1):120-5.

35. Kraemer SM, Kyes SA, Aggarwal G, Springer AL, Nelson SO, Christodoulou $Z$, et al. Patterns of gene recombination shape var gene repertoires in Plasmodium falciparum: comparisons of geographically diverse isolates. BMC Genomics. 2007;8:45

36. Molina-Cruz A, Zilversmit MM, Neafsey DE, Hartl DL, Barillas-Mury C. Mosquito vectors and the globalization of Plasmodium falciparum malaria. Annu Rev Genet. 2016:50(1):447-65.

37. Volkman SK, Sabeti PC, DeCaprio D, Neafsey DE, Schaffner SF, Milner DA, et al. A genome-wide map of diversity in Plasmodium falciparum. Nat Genet. 2007;39(1):113-9.

38. Manske M, Miotto O, Campino S, Auburn S, Almagro-Garcia J, Maslen G, et al. Analysis of Plasmodium falciparum diversity in natural infections by deep sequencing. Nature. 2012:487(7407):375-9.

39. Reilly Ayala HB, Wacker MA, Siwo G, Ferdig MT. Quantitative trait loci mapping reveals candidate pathways regulating cell cycle duration in Plasmodium falciparum. BMC Genomics. 2010;11(1):577.

40. Lambrechts L, Halbert J, Durand P, Gouagna LC, Koella JC. Host genotype by parasite genotype interactions underlying the resistance of anopheline mosquitoes to Plasmodium falciparum. Malar J. 2005;4:3.

41. Niaré O, Markianos K, Volz J, Oduol F, Touré A, Bagayoko M, et al. Genetic loci affecting resistance to human malaria parasites in a West African mosquito vector population. Science. 2002;298(5591):213-6.

42. Molina-Cruz A, DeJong RJ, Ortega C, Haile A, Abban E, Rodrigues J, et al. Some strains of Plasmodium falciparum, a human malaria parasite, evade the complement-like system of Anopheles gambiae mosquitoes. Proc Natl Acad Sci USA. 2012;109(28):E1957-62.

43. Singh P, Dhiman RC. Sporogonic cycles calculated using degree-days, as a basis for comparison of malaria parasite development in different ecoepidemiological settings in India. Jpn J Infect Dis. 2016;69(2):87-90.
44. Goodman CD, Siregar JE, Mollard V, Vega-Rodríguez J, Syafruddin D, Matsuoka $\mathrm{H}$, et al. Parasites resistant to the antimalarial atovaquone fail to transmit by mosquitoes. Science. 2016;352(6283):349-53.

45. Mharakurwa S, Kumwenda T, Mkulama MAP, Musapa M, Chishimba S, Shiff CJ, et al. Malaria antifolate resistance with contrasting Plasmodium falciparum dihydrofolate reductase (DHFR) polymorphisms in humans and Anopheles mosquitoes. Proc Natl Acad Sci USA. 2011;108:18796-801.

46. Sinka ME, Bangs MJ, Manguin S, Rubio-Palis Y, Chareonviriyaphap T, Coetzee M, et al. A global map of dominant malaria vectors. Parasit Vectors. 2012;5(1):69.

47. Cirimotich CM, Dong Y, Garver LS, Sim S, Dimopoulos G. Mosquito immune defenses against Plasmodium infection. Dev Comp Immunol. 2010;34(4):387-95.

48. Christophides GK, Vlachou D, Kafatos FC. Comparative and functional genomics of the innate immune system in the malaria vector Anopheles gambiae. Immunol Rev. 2004;198(1):127-48.

49. White RS, Rao W. Regulation of the control of Anopheles of the fluviatilisgroup by anti-adult spraying. Ind Med Gaz. 1944;79(8):364-9.

50. Ye YH, Chenoweth SF, Carrasco AM, Allen SL, Frentiu FD, van den Hurk AF, et al. Evolutionary potential of the extrinsic incubation period of dengue virus in Aedes aegypti. Evolution. 2016;70(11):2459-69.

51. Riehle MM, Guelbeogo WM, Gneme A, Eiglmeier K, Holm I, Bischoff E, et al. A cryptic subgroup of Anopheles gambiae is highly susceptible to human malaria parasites. Science. 2011;331(6017):596-8.

52. Alout H, Ndam NT, Sandeu MM, Djegbe I, Chandre F, Dabire RK, Djogbenou LS, Corbel V, Cohuet A. Insecticide resistance alleles affect vector competence of Anopheles gambiae s.s. for Plasmodium falciparum field isolates. PLoS One. 2013;8(5):e63849.

53. Joy DA, Gonzalez-Ceron L, Carlton JM, Gueye A, Fay M, McCutchan TF, Su X. Local adaptation and vector-mediated population structure in Plasmodium vivax malaria. Mol Bio Evo. 2008;25(6):1245-52.

54. Lefèvre T, Ohm J, Dabiré KR, Cohuet A, Choisy M, Thomas MB, et al. Transmission traits of malaria parasites within the mosquito: genetic variation, phenotypic plasticity, and consequences for control. Evol Appl. 2017:1-33.

55. Eling W, Hooghof J, van de Vegte-Bolmer M, Sauerwein R, van Gemert GJ. Tropical temperatures can inhibit development of the human malaria parasite Plasmodium falciparum in the mosquito. Proc Exp App Ent. 2001;12:1-6.

56. Vaughan JA, Noden BH, Beier JC. Population dynamics of Plasmodium falciparum sporogony in laboratory-infected Anopheles gambiae. J Parasitol. 1992;78(4):716-24.

57. Hien DFDS, Dabiré KR, Roche B, Diabaté A, Yerbanga RS, Cohuet $A$, et al. Plant-mediated effects on mosquito capacity to transmit human malaria. PLoS Pathog. 2016;12(8):e1005773.

58. Kligler IJ, Mer G. Studies on the effect of various factors on the infection rate of Anopheles elutus with different species of Plasmodium. Ann Trop Med Parasit. 1937;31(1):71

59. Lambrechts L, Chavatte J-M, Snounou G, Koella JC. Environmental influence on the genetic basis of mosquito resistance to malaria parasites. Proc $\mathrm{R}$ Soc B: Bio Sc. 2006;273(1593):1501-6.

60. Shapiro LLM, Murdock CC, Jacobs GR, Thomas RJ, Thomas MB. Larval food quantity affects the capacity of adult mosquitoes to transmit human malaria. Proc Biol Sci. 2016;283(1834):20160298.

61. Moller-Jacobs LL, Murdock CC, Thomas MB. Capacity of mosquitoes to transmit malaria depends on larval environment. Parasit Vectors. 2014:7:593.

62. Barreaux A, Barreaux P, Thievent K, Koella JC. Larval environment influences vector competence of the malaria mosquito Anopheles gambiae. Malaria World J. 2016;7(8):1-6.

63. Bara J, Rapti Z, Cáceres CE, Muturi EJ. Effect of larval competition on extrinsic incubation period and vectorial capacity of Aedes albopictus for dengue virus. PLoS One. 2015;10(5):e0126703.

64. Stone CM, Foster WA. Plant-sugar feeding and vectorial capacity. In: Takken W, Koenraadt CJM, editors. Ecology of parasite-vector interactions. Wageningen: Wageningen Academic Publishers; 2013. p. 35-79.

65. Armstrong PM, Ehrlich H, Bransfield A, Warren JL, Pitzer VE, Brackney DE. Successive bloodmeals enhance virus dissemination within mosquitoes and increase transmission potential. bioRxiv. 2018; https://doi.org/10.1101/246303.

66. Blanford S, Chan BHK, Jenkins N, Sim D, Turner RJ, Read AF, et al. Fungal pathogen reduces potential for malaria transmission. Science. 2005; 308(5728):1638-41.

67. Bargielowski I, Koella JC. A possible mechanism for the suppression of Plasmodium berghei development in the mosquito Anopheles gambiae by the microsporidian Vavraia culicis. PLoS One. 2009;4(3):e4676-5. 
68. Aliota MT, Chen C-C, Dagoro H, Fuchs JF, Christensen BM. Filarial worms reduce Plasmodium infectivity in mosquitoes. PLoS Negl Trop Dis. 2011;5(2):e963.

69. Cirimotich CM, Ramirez IL, Dimopoulos G. Native microbiota shape insect vector competence for human pathogens. Cell Host Microbe. 2011;10(4):307-10.

70. Pollitt LC, Churcher TS, Dawes EJ, Khan SM, Sajid M, Basáñez M-G, et al. Costs of crowding for the transmission of malaria parasites. Evol Appl. 2013;6(4):617-29.

71. Pollitt LC, Bram JT, Blanford S, Jones MJ, Read AF. Existing infection facilitates establishment and density of malaria parasites in their mosquito vector. PLoS Pathog. 2015;11(7):e1005003.

72. Imwong M, Nakeesathit S, Day NPJ, White NJ. A review of mixed malaria species infections in anopheline mosquitoes. Malar J. 2011;10(1):253.

73. Putri YE, Rozi S, Tasman H, Aldila D. Assessing the effect of extrinsic incubation period (EIP) prolongation in controlling dengue transmission with Wolbachia-infected mosquito intervention. AlP Conference Proceedings. 2017:020019.

74. Carrington LB, Tran BCN, Le NTH, Luong TTH, Nguyen TT, Nguyen PT, et al. Field- and clinically-derived estimates of Wolbachia-mediated blocking of dengue virus transmission potential in Aedes aegypti mosquitoes. Proc Natl Acad Sci USA. 2018;115(2):361-6.

75. Lorenz LM, Koella JC. Maternal environment shapes the life history and susceptibility to malaria of Anopheles gambiae mosquitoes. Malar J. 2011; 10(1):382.

76. Vantaux A, Dabiré KR, Cohuet A, Lefèvre T. A heavy legacy: offspring of malaria-infected mosquitoes show reduced disease resistance. Malar J. 2014;13:442.

77. Paaijmans KP, Blanford S, Chan BHK, Thomas MB. Warmer temperatures reduce the vectorial capacity of malaria mosquitoes. Biol Lett. 2012;8(3):465-8.

78. Hay SI, Cox J, Rogers DJ, Randolph SE, Stern DI, Shanks GD, et al. Climate change and the resurgence of malaria in the East African highlands. Nature. 2002;415(6874):905-9.

79. Pascual M, Ahumada JA, Chaves LF, Rodó X, Bouma M. Malaria resurgence in the East African highlands: temperature trends revisited. Proc Natl Acad Sci USA. 2006;103(15):5829-34.

80. Gething PW, Smith DL, Patil AP, Tatem AJ, Snow RW, Hay SI. Climate change and the global malaria recession. Nature. 2010;465(7296):342-5.

81. Paaijmans KP, Blanford Jl, Crane RG, Mann ME, Ning L, Schreiber KV, et al. Downscaling reveals diverse effects of anthropogenic climate warming on the potential for local environments to support malaria transmission. Climatic Change. 2014;125(3-4):479-88.

82. Siraj AS, Santos-Vega M, Bouma MJ, Yadeta D, Carrascal DR, Pascual M. Altitudinal changes in malaria incidence in highlands of Ethiopia and Colombia. Science. 2014;343(6175):1154-8.

83. Parham PE, Waldock J, Christophides GK, Hemming D, Agusto F, Evans K, et al. Climate, environmental and socio-economic change: weighing up the balance in vector-borne disease transmission. Philos Trans R Soc Lond B Biol Sci. 2015;370(1665):20130551.

84. Ye YH, Carrasco AM, Frentiu FD, Chenoweth SF, Beebe NW, van den Hurk $A F$, et al. Wolbachia reduces the transmission potential of dengue-infected Aedes aegypti. PLoS Negl Trop Dis. 2015;9(6):e0003894.

85. Fontaine A, Jiolle D, Moltini-Conclois I, Lequime S, Lambrechts L. Excretion of dengue virus RNA by Aedes aegypti allows non-destructive monitoring of viral dissemination in individual mosquitoes. Sci Rep. 2016;6:24885.

86. Rund S, O'Donnell A, Gentile J, Reece S. Daily rhythms in mosquitoes and their consequences for malaria transmission. Insects. 2016;7(2):14-20.

87. Cator LJ, Pietri JE, Murdock CC, Ohm JR, Lewis EE, Read AF, et al. Immune response and insulin signalling alter mosquito feeding behaviour to enhance malaria transmission potential. Sci Rep. 2015;5:11947.

88. Ohm JR, Teeple J, Nelson WA, Thomas MB, Read AF, Cator LJ. Fitness consequences of altered feeding behavior in immune-challenged mosquitoes. Parasit Vectors. 2016;9(1):113.

89. Greischar MA, Read AF, Bjørnstad ON. Synchrony in malaria infections: How intensifying within-host competition can be adaptive. Am Nat. 2014;183(2):E36-49.

90. Mideo N, Reece SE, Smith AL, Metcalf JE. The Cinderella syndrome: why do malaria infected cells burst at midnight? Trends Parasitol. 2013;29(1):10-6.

91. Koella JC, Lynch PA, Thomas MB, Read AF. Towards evolution-proof malaria control with insecticides. Evol Appl. 2009;2(4):469-80.

92. Read AF, Lynch PA, Thomas MB. How to make evolution-proof insecticides for malaria control. PLoS Bio. 2009; 7(4):1000058.

93. Sternberg ED, Thomas MB. Insights from agriculture for the management of insecticide resistance in disease vectors. Evol Appl. 2017;11(1786):164-11.

\section{Submit your next manuscript to BioMed Central and we will help you at every step:}

- We accept pre-submission inquiries

- Our selector tool helps you to find the most relevant journal

- We provide round the clock customer support

- Convenient online submission

- Thorough peer review

- Inclusion in PubMed and all major indexing services

- Maximum visibility for your research

Submit your manuscript at www.biomedcentral.com/submit 